\title{
Determination of the mechanism of action of repetitive halothane exposure on rat brain tissues using a combined method of microarray gene expression profiling and bioinformatics analysis
}

\author{
JIANSHENG WANG, XIAOJUN YANG, HUAN XIAO, JIANQIANG KONG and MIAO BING
}

\begin{abstract}
Department of Anesthesiology, Shanghai Baoshan District Integrated Traditional and Western Medicine Hospital, Shanghai 201900, P.R. China
\end{abstract}

Received July 30, 2014; Accepted April 15, 2015

DOI: $10.3892 / \mathrm{mmr} .2015 .4462$

\begin{abstract}
The present study aimed to investigate the gene expression profiles of rats brain tissues treated with halothane compared with untreated controls to improve current understanding of the mechanism of action of the inhaled anesthetic. The GSE357 gene expression profile was dowloaded from the Gene Expression Omnibus database, and included six gene chips of samples repeatedly exposed to halothane and 12 gene chips of untreated controls. The differentially expressed genes (DEGs) between these two groups were identified using the Limma package in R language. Subsequently, the Database for Annotation, Visualization and Integrated Discovery was used to annotate the function of these DEGs. In addition, the most significantly upregulated gene and downregulated gene were annotated, to reveal the functional interactions with other associated genes, in FuncBase database. A total of 44 DEGs were obtained between The control and halothane exposure samples. Following Gene Ontology functional classification, these DEGs were found to be involved predominantly in the circulatory system, regulation of cell proliferation and response to endogenous stimulus and corticosteroid stimulus processes. KRT31 and HMGCS2, which were identified as the most significantly downregulated and upregulated DEGs, respectively, were associated with the lipid metabolic process and T cell activation, respectively. These results provided a basis for the development of improved inhalational anesthetics with minimal side effects and are essential for optimization of inhaled anesthetic techniques for advanced surgical procedures.
\end{abstract}

Correspondence to: Dr Jiansheng Wang, Department of Anesthesiology, Shanghai Baoshan District Integrated Traditional and Western Medicine Hospital, 181 Youyi Road, Baoshan, Shanghai 201900, P.R. China

E-mail: jianshengwdr@163.com

Key words: halothane repetitive exposure, differentially expressed genes, gene function, DNA microarray

\section{Introduction}

According to statistical reports, there are $>200,000,000$ individuals worldwide requiring anesthetic care for surgery each year (1). Anesthetics exert the three reversible characteristics of immobility, amnesia and unconsciousness (2). General anesthetic drugs include inhaled gases and intravenous agents, which can cause a reduction in nerve transmission at synapses (3). Halothane, an inhaled anesthetic, is partly metabolized by the liver and the metabolized products are excreted in the urine $(4,5)$. Previous associated studies have indicated that repeated exposure to halothane in adults may result in halothane-associated liver failure (6-8).

Previous molecular investigations have provided evidence that the mechanism of volatile anesthetic involves a series of molecular modulation. The actions of anesthetics lies within the family of ligand-gated ion channels and the binding of anesthetics may alter the overall motion of a ligand-gated ion channel (9). The ligand-gated ion channel superfamily contains $\mathrm{GABA}_{\mathrm{A}}$ receptors, glycine receptors, serotonin type 3 receptors and nicotinic acetylcholine receptors. $\mathrm{GABA}_{\mathrm{A}}$ receptors, neurotransmitter-gated chloride channels, are located on neurons and, when activated, they reduce neuronal excitation (10). Protein kinase C, a soluble cytoplasmic protein, is an important signal transduction enzyme, which is involved in regulating the release of neurotransmitters and ion channel activity $(11,12)$. The results of a study by Maingret et al demonstrated that TREK-1, a two-pore-domain background postassium channel, can be activated by volatile anesthetics and was suggested to be a target in the action of these drugs (13) Associated investigations have reported that the general mechanism of halothane may be associated with competition with endogenous ligands, and mitochondrion are a preferred and saturable site for halothane localization (14). In addition, previous analysis of brain membrane proteins in rats has revealed selective binding of halothane to individual protein subunits of the mitochondrial respiratory chain (15). However, these findings regarding the mechanism of action of volatile anesthetics are limited. Advancements in microarray technology have assisted in providing a comprehensive analysis for gene expression among anesthetics (16). It provides a 
useful tool for the identification of featured genes associated with anesthetic action.

In the present study, a set of gene expression profiles, including unexposed controls and those of exposure to halothane were used to analyze their differentially expressed genes (DEGs). Subsequently, bioinformatics tools were used to identify the functions associated with these DEGs. The aim of this investigation was to identify specific genes involved in the action of anesthetics. The results may assist in providing a more profound understanding of the molecular mechanism of anesthetics and in overcoming the adverse effects arising from their use.

\section{Materials and methods}

Affymetrix microarray data. Microarray data from the Gene Expression Omnibus (GEO) database were downloaded under the accession number, GSE357, which were deposited by the University of Pennsylvania (Pennsylvania, USA) (17). A total of 18 gene chips were available. The 18 specimens included 12 control specimens and six repeated halothane exposure specimens. The experiment protocol used by the University of Pennsylvania to obtain these data is briefly described as follows: Rats ( $\mathrm{n}=18$; male; weigh, $250 \mathrm{~g}$ ) were divided into either an unexposed control group $(n=12)$ or a repetitive exposure group $(n=6)$. Each of the rats in the repetitive exposure group was exposed to $0.8 \%$ halothane each day for $90 \mathrm{~min}$, twice daily (with $3 \mathrm{~h}$ recovery between exposures) for 2.5 or 5 days, for a total of five or 10 exposures, respectively. None of the animals required intubation. The microarray expression platform was termed GPL85 [RG_U34A] Affymetrix Rat Genome U34 Array. In the present study, the original data was downloaded as well as the annotation of platform.

Data preprocessing and analysis of DEGs. The data in the CEL files were converted into expression profiles using the Affy package (http://www.bioconductor. org/help/search/index.html?q=Affy, Affymetrix,Santa Clara, California, US) in R (18) and data were normalized using the median algorithm. The Limma package (http://www.bioconductor.org/packages/release/bioc/html/limma.html) in $\mathrm{R}$ language was used to analyze the DEGs between the 12 control samples and six exposure samples (19). A P-value $<0.1$ and a $\log \mathrm{FCl}$-value $>1$ were set as the threshold criteria for DEGs.

Gene ontology (GO) enrichment analysis. The GO project (http://www.geneontology.org/) provides structured, controlled vocabularies and classifications, which encompass the three domains of cellular component, molecular function and biological process (20). The Database for Annotation, Visualization and Integrated Discovery (DAVID, http://david.abcc.ncifcrf.gov/) consists of an integrated biological knowledge-base and functional annotation charts or tables. It provides a comprehensive set of functional annotation tools for the integration of particular genes of interest with a specific function $(21,22)$. All the DEGs, which were identified in the present study using the Limma Package in $\mathrm{R}$ language, were loaded into the DAVID database, and a significant value was calculated for each of the GO terms
Table I. DEGs between the control and exposure groups, consisting of 19 downregulated genes and 25 upregulated genes.

\begin{tabular}{|c|c|c|}
\hline Gene & P-value & $\log \mathrm{FC}$ \\
\hline KRT31 & 0.0032 & -1.4436361 \\
\hline NOS3 & 0.00125 & -1.3555293 \\
\hline$C D C 25 B$ & 0.0134 & -1.3488796 \\
\hline VOM $2 R 32$ & 0.00813 & -1.2816925 \\
\hline$H M G B 1$ & 0.00994 & -1.2430645 \\
\hline$R A B 33 B$ & 0.00354 & -1.2112175 \\
\hline PEX10 & 0.0026 & -1.2037957 \\
\hline PAM & 0.00907 & -1.1834612 \\
\hline B3GNTL1 & 0.0126 & -1.1714498 \\
\hline GPX3 & 0.0076 & -1.1710799 \\
\hline FCERIA & 0.0413 & -1.1358269 \\
\hline TPH1 & 0.0219 & -1.1336363 \\
\hline GLPIR & 0.00643 & -1.1331447 \\
\hline$M T R$ & 0.0015 & -1.1152652 \\
\hline АCTA2 & 0.0252 & -1.0915312 \\
\hline VOM1R101 & 0.0103 & -1.0725745 \\
\hline$H A P 1$ & 0.00347 & -1.0706768 \\
\hline IGSF6 & 0.0152 & -1.0437975 \\
\hline$D D X 4$ & 0.0464 & -1.0081465 \\
\hline HIST1H2AF & 0.0248 & 1.0072684 \\
\hline$U B E 2 D 4$ & 0.0226 & 1.0169088 \\
\hline ACOT1 & 0.0229 & 1.0187761 \\
\hline MMP11 & 0.0167 & 1.0214673 \\
\hline$A L B$ & 0.0201 & 1.0385379 \\
\hline$R G S 1$ & 0.0498 & 1.0405889 \\
\hline OLR1496 & 0.0198 & 1.0563126 \\
\hline UGT2B15 & 0.0469 & 1.0583942 \\
\hline$K L K 1 C 3$ & 0.0173 & 1.077667 \\
\hline$T M I G D 1$ & 0.00601 & 1.0904489 \\
\hline$T S X$ & 0.0229 & 1.1425503 \\
\hline CNGA3 & 0.00984 & 1.1530625 \\
\hline$I G F 2 R$ & 0.000198 & 1.2007601 \\
\hline NOX4 & 0.0378 & 1.218972 \\
\hline PCSK5 & 0.0338 & 1.2191295 \\
\hline$L H X 1$ & 0.0124 & 1.2359783 \\
\hline TLR4 & 0.00922 & 1.2781933 \\
\hline RNF4 & 0.0198 & 1.3328296 \\
\hline SLC1A6 & 0.0191 & 1.3830303 \\
\hline ZMAT3 & 0.0122 & 1.4563084 \\
\hline NXT1 & 0.00583 & 1.4660187 \\
\hline OPA1 & 0.00332 & 1.7659469 \\
\hline FВXО30 & 0.0258 & 1.7748347 \\
\hline$L E P R$ & 0.00319 & 2.1167477 \\
\hline$H M G C S 2$ & 0.00005 & 2.2001709 \\
\hline
\end{tabular}

Thresholds were set at $\mathrm{P}<0.05$ and $\log \mathrm{FC}<1$ (downregulation) and $\log \mathrm{FC}>1$ (upregulated). DEG, differentially expressed gene.

identified. A count number $>2$ and a false discovery rate $($ FDR $)<0.05$ were selected as the cut-off criteria. 


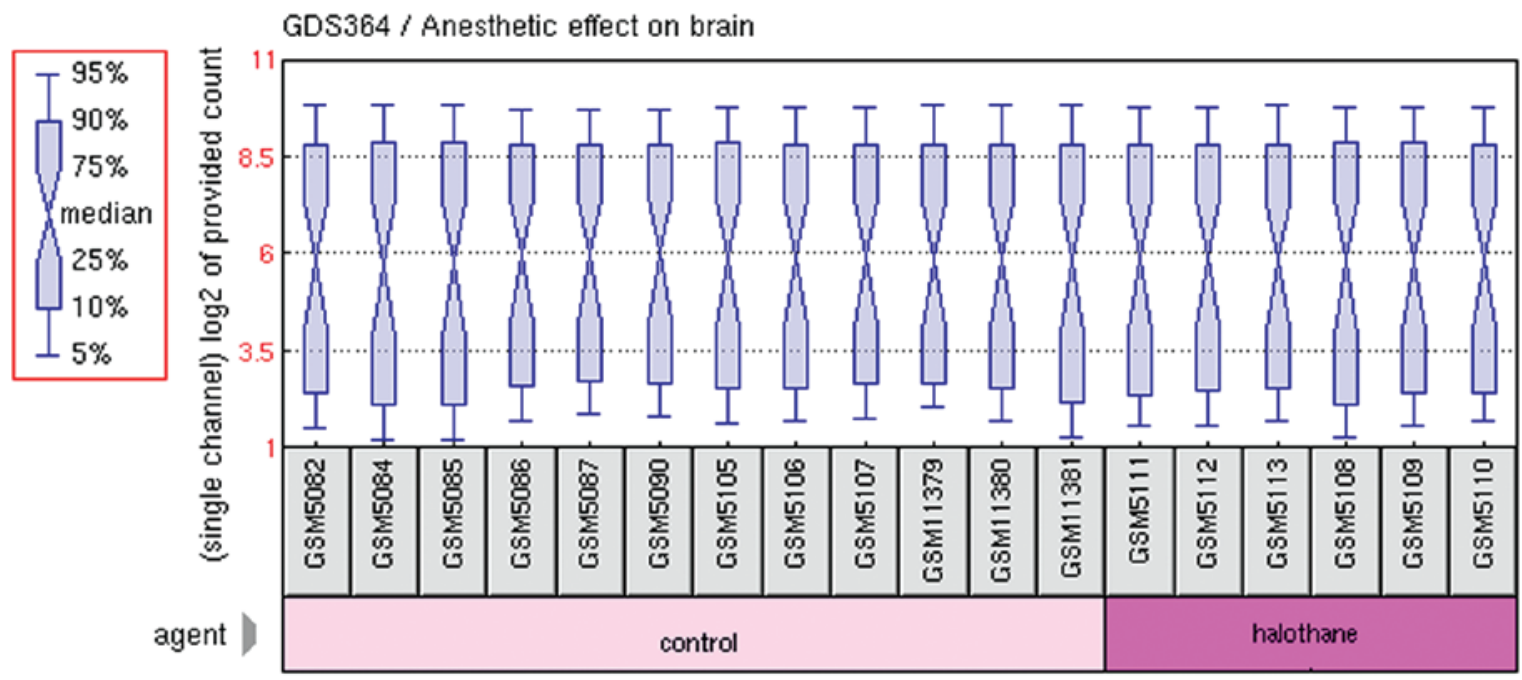

Figure 1. Box-whisker plot to analyze the normalization of gene expression data. The light pink area indicates the gene expression in the control rats brain tissue and the dark pink area indicates gene expression in the halothane-exposed rats brain tissue treated by halothane. The horizontal black line in centre of box is indicates the median value of expression. The quality of gene expression data can be determined by the distribution of the data either side of the black line. The results demonstrated that the median value was located at the same level.

Network analysis. FuncBase is a web resource for viewing quantitative machine learning-based gene function annotations (http://func.mshri.on.ca/) (23). Predictions in FuncBase can be viewed using $\mathrm{GO}$ terms. In the present study, the FuncBase database was used to annotate the functional interactions between the significantly DEGs and other genes by calculating their score, which, for the GO function node was based on the number of genes of similar function $(24,25)$. The records with scores $>0.8$ were retained.

\section{Results}

Screening of DEGs. In the present study, the publicly available GSE357 microarray dataset was obtained from the GEO database. Following data preprocessing and normalization (Fig. 1), the data were analyzed using Limma package in $\mathrm{R}$ language to identify the DEGs between the 12 control and six exposure samples. According to the threshold criterion $(\mathrm{P}<0.05$ and $\log \mathrm{FCl}>1)$ for DEGs, a total of 44 DEGs were obtained, consisting of 19 downregulated and 25 upregulated genes (Table I). Subsequently, the genes exhibiting the most significant upregulation and downregulation were selected, which were HMGCS2 $(\mathrm{P}=0.00005)$ and KRT31 $(\mathrm{P}=0.0032)$, respectively.

GO enrichment analysis of the DEGs. In order to investigate the expression of the DEGs in the exposure group at a more functional level, the DEGs $(\mathrm{P}<0.05$ and $\log \mathrm{FCl}>1)$ between the control and exposure profiles were classified into $\mathrm{GO}$ terms (Table II). A count number $>2$ and FDR $<0.05$ were selected as the cut-off criteria. All the DEGs were enriched in $18 \mathrm{GO}$ terms, including response to steroid hormone and endogenous stimulus. GO terms were also associated with metabolic and circulatory system processes.

Interaction network construction. The present study used the FuncBase database to annotate the functional interactions between the significantly DEGs and other genes, and screened for significant interactions with a score $>0.8$. By integrating these associations, interaction networks of these two significant DEGs and their interactive genes were constructed (Fig. 2). The database revealed gene function nodes in two networks from this database, KRT31 and HMGCS2, expressing similar function genes in four function nodes and five function nodes, respectively (Tables III and IV). The function nodes with the highest scores were associated with lipid metabolism (GO: 6629) and T cell activation (GO: 42110).

\section{Discussion}

In the present study, the effects of halothane on the brain tissue of rats were investigated. The results demonstrated that halothane modulated the expression of 44 DEGs, which were involved predominantly in response to endogenous and corticosteroid stimuli.

The results of the present study described the differential gene expression profiling between unexposed control samples and halothane exposure samples. Certain effects of halothane on regulated genes (HMGB1, TLR4, HMGCS2) were involved in responding to hormone and endogenous stimuli. HMGCS2, was one of the most markedly upregulated genes in tyhe exposure profile compared with the control. The role of this gene in the anesthetic mechanism remains to be fully elucidate, however, HMGCS2 has been demonstrated to correlate with fatty acid metabolism (26). Toll-like receptor 4 (TLR4) is a member of the TLR family, which are pattern recognition receptors that can activate the innate immune response (27). High mobility group box-1 (HMGB1), an endogenous danger signal, is released from injured cells and signals inflammatory responses by binding to pattern recognition receptors, including TLR4 (28). It has been demonstrated that intracellular TFA adducts, metabolized by halothane, can induce a stress response in hepatocytes and activate associated innate immune cells (29). Then activated immune cells release cytotoxic granules leading 


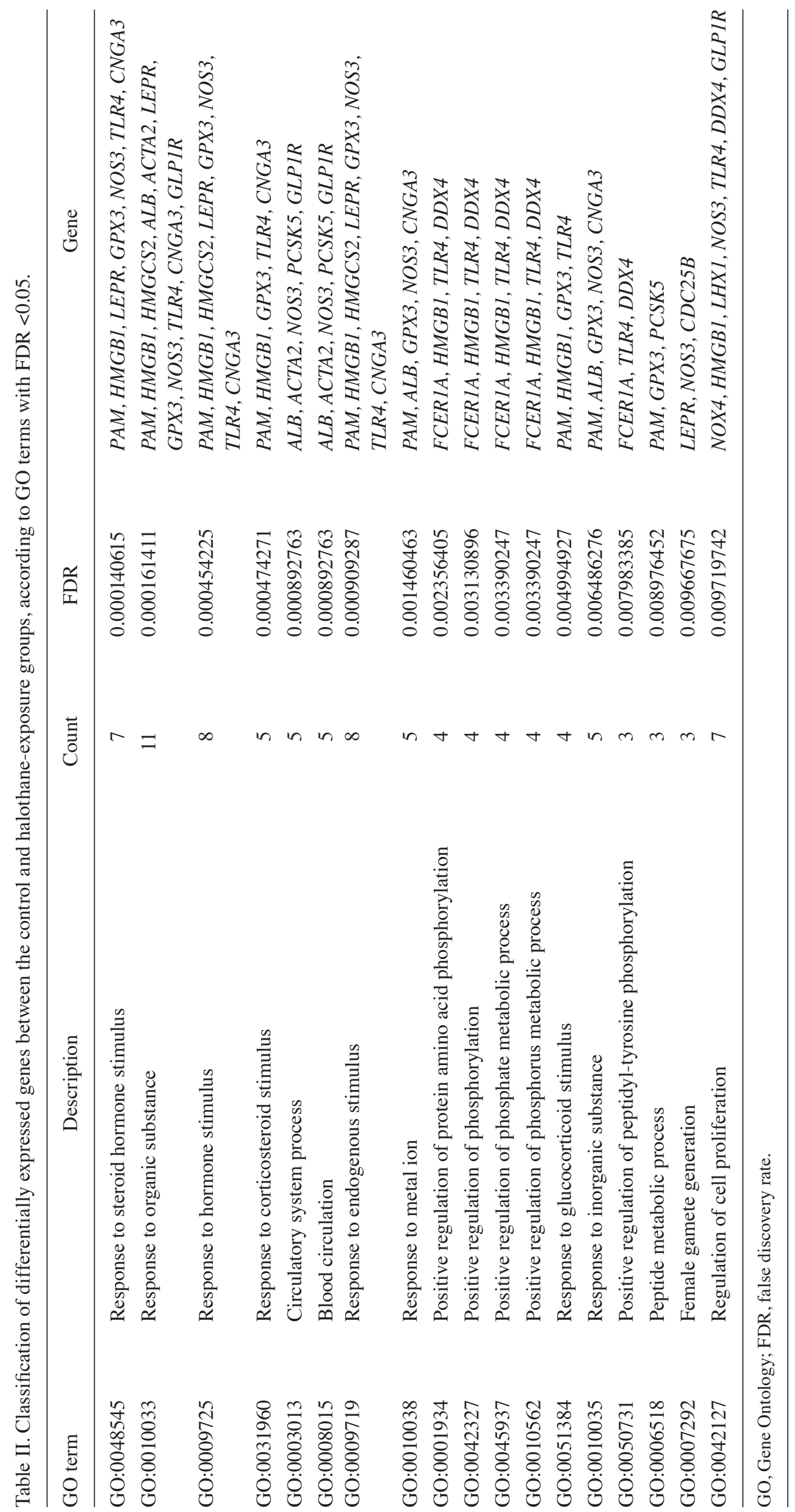


Table III. Function node scores of KRT31 in the network.

\begin{tabular}{lll}
\hline GO term & \multicolumn{1}{c}{ Description } & Score \\
\hline GO: 6629 & Lipid metabolic process & 0.970 \\
GO: 44255 & Cellular lipid metabolic process & 0.954 \\
GO: 19752 & Carboxylic acid metabolic process & 0.866 \\
GO: 6082 & Organic acid metabolic process & 0.846
\end{tabular}

GO, Gene Ontology.

Table IV. Function node scores of HMGCS2 in the network.

\begin{tabular}{llc}
\hline GO term & \multicolumn{1}{c}{ Description } & Score \\
\hline GO: 42110 & T cell activation & 0.898 \\
GO: 42098 & T cell proliferation & 0.836 \\
GO: 9913 & Epidermal cell differentiation & 0.826 \\
GO: 8544 & Epidermis development & 0.808 \\
GO: 42129 & Regulation of T cell proliferation & 0.806 \\
\hline
\end{tabular}

GO, Gene Ontology.

to hepatocellular necrosis. Meanwhile, $H M G B-1$ acts as a TLR4 agonist to enhance HAL-induced liver injury (29). It also has been reported that $H M G B-1$ may be part of a sexually dimorphic innate immune response in halothane-treated mice (30), which may be consistent with the involvement of $H M G B-1$ in response to hormone stimuli. $H M G B-1$ is also involved in responding to corticosteroid stimuli. A previous study revealed that glucocorticoids, which act via glucocorticoid receptor (GR) to regulate target gene transcription, may control metabolic energy in hepatic processes (31). There exists a physical interaction between $H M G B-1$ and GR (32). The present study hypothesized that $H M G B-1$ may be regulated by glucocorticoids in hepatic processes. KRT31, a member of the keratin gene family, was significantly downregulated in the exposure profile, compared with the control profile. It has been reported that KRT31 is essential for the maintenance of hepatocyte structural and functional integrity (33). Halothane has been demonstrated to induce liver injury and halothane hepatitis (29), and this evidence may account for the downregulation of KRT31 following halothane exposure. In addition, the functional enrichment analysis of the KRT31-centered network suggested the biological process of lipid metabolism was dysregulated following halothane exposure. This result is consistent with that of a previous study, which suggested that keratin polypeptides, obtained from mice, were modified by the covalent attachment of lipids (34). Therefore, the present study hypothesized that the involvement of KRT31 in lipid metabolism may be dysfunctional under halothane exposure.

In conclusion, the data obtained in the present study provided a comprehensive bioinformatics analysis of genes and networks which may be involved in the effect of inhaled anesthetis. A total of 44 DEGs were identified from the

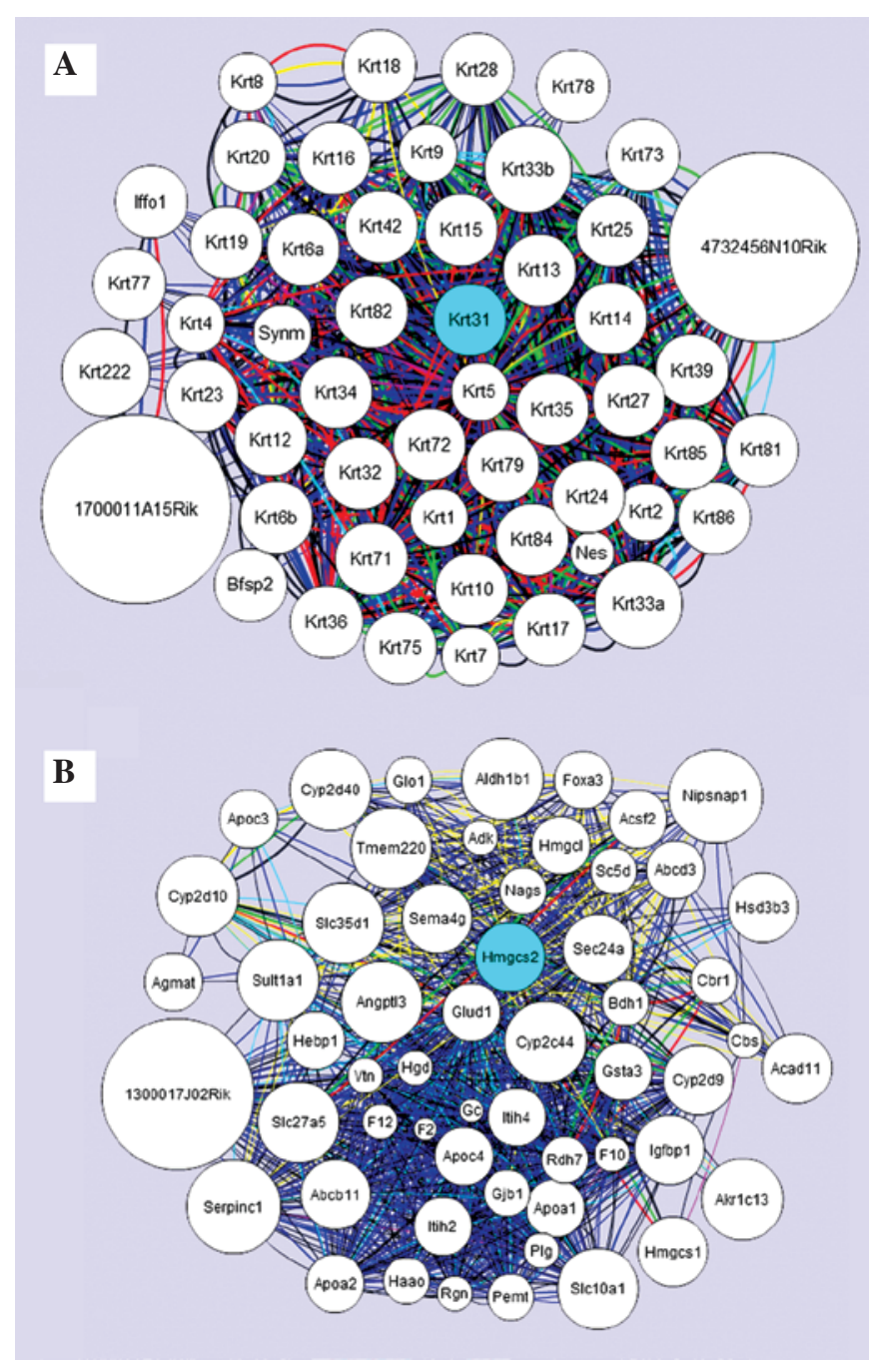

Figure 2. (A) Interaction network constructed in the halothane-exposure group, containing the downregulated KRT31 gene. (B) Interaction network constructed in the halothane-exposure group, containing the upregulated $H M G C S 2$ gene. The blue nodes indicate the differentially expressed genes and the white nodes indicate their interactive genes.

GSE357 accession. Furthermore, thee results of the present study demonstrated that genes, including $H M G B-1$ and TLR4 may be important in the occurrence of halothane-induced hepatotoxicity, and KRT31 may be closely associated with lipid metabolism in the liver. These DEGs may be used as specific therapeutic molecular targets in liver failure. However, there have been no reports on the expression of $H M G C S 2$ in the immune response, therefore, its potential role in anesthetics remains to be elucidated. It may be a specific biomarker in the mechanism of inhaled anesthetics in the brain. Following these observations, further investigations are required to more closely investigate the anesthetic effect, which genes present.

\section{References}

1. Weiser TG, Regenbogen SE, Thompson KD, et al: An estimation of the global volume of surgery: a modelling strategy based on available data. Lancet 372: 139-144, 2008.

2. Eger EI 2nd and Sonner JM: Anaesthesia defined (gentlemen, this is no humbug). Best Pract Res Clin Anaesthesiol 20: 23-29, 2006. 
3. Richter J, Landau EM and Cohen S: The action of volatile anesthetics and convulsants on synaptic transmission: A unified concept. Mol Pharmacol 13: 548-559, 1977.

4. Alkire MT, Pomfrett CJ, Haier RJ, et al: Functional brain imaging during anesthesia in humans: effects of halothane on global and regional cerebral glucose metabolism. Anesthesiology 90: 701-709, 1999.

5. MatsumotoN,KoizumiMand SugaiM:Hepatolobectomy-induced depression of hepatic circulation and metabolism in the dog is counteracted by isoflurane, but not by halothane. Acta Anaesthesiol Scand 43: 850-854, 1999.

6. Harrfeldt HP: Acute liver failure following repeated halothane anesthesia-related to an accident covered by industrial insurance. Monatsschr Unfallheilkd Versicher Versorg Verkehrsmed 72: 317, 1969 (In German).

7. Lo SK, Wendon J, Mieli-Vergani G and Williams R: Halothane-induced acute liver failure: continuing occurrence and use of liver transplantation. Eur J Gastroenterol Hepatol 10 635-639, 1998

8. Wessler S: Halothane and liver failure. JAMA 213: 129, 1970.

9. Hemmings HC Jr, Akabas MH, Goldstein PA, Trudell JR, Orser BA and Harrison NL: Emerging molecular mechanisms of general anesthetic action. Trends Pharmacol Sci 26: 503-510, 2005.

10. Nishikawa K, Jenkins A, Paraskevakis I and Harrison NL: Volatile anesthetic actions on the GABAA receptors: contrasting effects of alpha 1 (S270) and beta 2 (N265) point mutations. Neuropharmacology 42: 337-345, 2002.

11. Gomez RS, Guatimosim C and Gomez MV: Mechanism of action of volatile anesthetics: role of protein kinase C. Cell Mol Neurobiol 23: 877-885, 2003.

12. Hemmings $\mathrm{HC} \mathrm{Jr}$ and Adamo AI: Effects of halothane and propofol on purified brain protein kinase $\mathrm{C}$ activation. Anesthesiology 81: 147-155, 1994

13. Maingret F, Patel AJ, Lesage F, Lazdunski M and Honoré E: Mechano- or acid stimulation, two interactive modes of activation of the TREK-1 potassium channel. J Biol Chem 274 26691-26696, 1999.

14. Eckenhoff RG and Shuman H: Localization of volatile anesthetic molecules at the subcellular and molecular level. Ann N Y Acad Sci 625: 755-756, 1991.

15. Xi J, Liu R, Asbury GR, Eckenhoff MF and Eckenhoff RG: Inhalational anesthetic-binding proteins in rat neuronal membranes. J Biol Chem 279: 19628-19633, 2004.

16. Chepovetsky J, Kalir T and Weiderpass E: Clinical applicability of microarray technology in the diagnosis, prognostic stratification, treatment and clinical surveillance of cervical adenocarcinoma. Curr Pharm Des 19: 1425-1429, 2013.

17. Pan JZ, Wei H, Hecker JG, Tobias JW, Eckenhoff RG and Eckenhoff MF: Rat brain DNA transcript profile of halothane and isoflurane exposure. Pharmacogenet Genomics 16: 171-182, 2006.

18. Xia X, Mcclelland M and Wang Y: WebArray: an online platform for microarray data analysis. BMC Bioinformatics 6: 306, 2005.
19. Fernandez P, Soria M, Blesa D, et al: Development, characterization and experimental validation of a cultivated sunflower (Helianthus annuus L.) gene expression oligonucleotide microarray. PLoS One 7: e45899, 2012.

20. Ashburner M, Ball CA, Blake JA, et al: Gene ontology: tool for the unification of biology. The Gene Ontology Consortium. Nat Genet 25: 25-29, 2000.

21. Brown DL and Brock-Utne JG: The first structure-function approach to drug design in anaesthesia. Dr David S. Savage and pancuronium bromide. Anaesthesia 52: 1202-1204, 1997.

22. Quentien MH, Delemer B, Papadimitriou DT, et al: Deficit in anterior pituitary function and variable immune deficiency (DAVID) in children presenting with adrenocorticotropin deficiency and severe infections. J Clin Endocrinol Metab 97: E121-E128, 2012.

23. Beaver JE, Tasan M, Gibbons FD, Tian W, Hughes TR and Roth FP: FuncBase: a resource for quantitative gene function annotation. Bioinformatics 26: 1806-1807, 2010.

24. Shakouri P, Ordys A and Askari MR: Adaptive cruise control with stop \& go function using the state-dependent nonlinear model predictive control approach. ISA Trans 51: 622-631, 2012.

25. Wang J, Zhou X, Zhu J, et al: GO-function: deriving biologically relevant functions from statistically significant functions. Brief Bioinform 13: 216-227, 2012.

26. Wright J, Morales MM, Sousa-Menzes J, et al: Transcriptional adaptation to Clen5 knockout in proximal tubules of mouse kidney. Physiol Genomics 33: 341-354, 2008.

27. Medzhitov R and Janeway C Jr: Innate immune recognition: mechanisms and pathways. Immunol Rev 173: 89-97, 2000.

28. Park JS, Svetkauskaite D, He Q, et al: Involvement of Toll-like receptors 2 and 4 in cellular activation by high mobility group box 1 protein. J Biol Chem 279: 7370-7377, 2004.

29. Dugan CM, Fullerton AM, Roth RA and Ganey PE: Natural killer cells mediate severe liver injury in a murine model of halothane hepatitis. Toxicol Sci 120: 507-518, 2011.

30. Scaffidi P, Misteli T and Bianchi ME: Release of chromatin protein HMGB1 by necrotic cells triggers inflammation. Nature 418: 191-195, 2002.

31. Nyirenda MJ, Lindsay RS, Kenyon CJ, Burchell A and Seckl JR: Glucocorticoid exposure in late gestation permanently programs rat hepatic phosphoenolpyruvate carboxykinase and glucocorticoid receptor expression and causes glucose intolerance in adult offspring. J Clin Invest 101: 2174-2181, 1998.

32. Agresti A, Scaffidi P, Riva A, Caiolfa VR and Bianchi ME: GR and HMGB1 interact only within chromatin and influence each other's residence time. Mol Cell 18: 109-121, 2005.

33. Loranger A, Duclos S, Grenier A, et al: Simple epithelium keratins are required for maintenance of hepatocyte integrity. Am J Pathol 151: 1673-1683, 1997.

34. Asch HL, Mayhew E, Lazo RO and Asch BB: Lipids covalently bound to keratins of mouse mammary epithelial cells. Biochem Mol Biol Int 29: 1161-1169, 1993. 